\title{
REVUE
}

\section{Quelques sous-produits alimentaires peu connus ou peu utilisés}

\author{
par M.G. CURASSON
}

Il est, en régions chaudes, divers sous-produits de l'industrie indigène ou européenne qui ne tiennent une place dans l'alimentation des animaux que dans des conditions limitées de lieu et d'utilisation. Il en est aussi qui, délaissés, pourraient être utilisés; d'autres dont la valeur est mal déterminée. Il nous a paru intércssant de passer en revue les principaux d'entre eux.

\section{I. - TOURTEAUX \\ Tourteau de Karité}

La « noix ». de Karité (Butyrospermum Parkii) fournit, après extraction de la matière grasse, un tourteau dont la teneur en protides varie entre 15 et $17 \%$, ce qui permettrait de l'utiliser comme aliment azoté. L'expérimentation, malheureusement, a été trop peu poussée pour qu'on en puisse tirer des conclusions. En effet, le seul essai tenté par Jacquot et Guillemet chez le rat a conduit les auteurs à dire que, en raison du taux exagéré en indigestible glucidique, son introduction dans la ration est cause d'un dangereux déséquilibre, et « perturbe l'utilisation digestive des rations les mieux équilibrées ». A $20 \%$ de la ration du rat, il cause un amaigrissement considérable; à plus forte dose, il est rapidement mortel.

\section{Tourteau de Kapolk}

Le tourteau provient de la graine de divers Kapokiers (Bombax buonopozenze) qui fournit 18 à $20 \%$ d'huile. Diversement apprécié, ce tourteau était surtout utilisé comme engrais et parfois pour l'alimentation du bètail, à condition qu'il ne renferme pas trop de fibres; on le considérait aussi comme suspect en raison de la présence de saponine.

Au cours de la dernière guerre, on a essayé le « kapok désamertumé » produit pulvérulent débarrassé des saponines. Les essais ont été réalisés chez le mouton (Leroy et Gasnier), chez le porc (Thiery), chez le lapin. Sa valeur nutritive s'est révélée très faible. Chez le rat, il se révèle dangereux ainsi que chez le lapin. Chez le porc, il provoque de phénomènes d'intoxication.

\section{Tourteau de Baobab}

La graine de baobab (Adansonia digitata) renferme une huile parfois consommée (Madagascar) et qu'on a tenté d'industrialiser. Le tourteau a eté recommandé pour l'alimentation du bétail, mais il ne semble pas qu'il soit entré dans la pratique. Sa composition est la suivante :

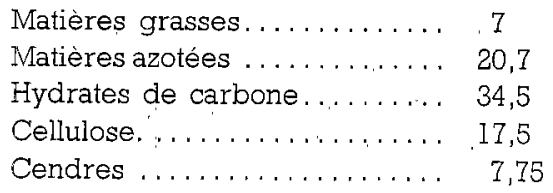

Tourteau de Moringa pterygosperma

Les graines de Moringa pterygosperma ( « ben » au Sénégal) donnent une huile couramment utilisée. Le tourteau a été recommandé pour les animaux. Cependant, il renferme une saponine. Sa composition comprend :

Matières grasses............ 14,5

Matières azotées............ 31

Hydrates de carbone......... 20

Cellulose. .............. 22

\section{Tourteau d'Aleurites}

Les divers Aleurites foumissent des graines dont on extrait des huiles industrielles. Les tourteaux sont de valeur inégale; celui d'A. moluccana peut être purgatif; ceux d'A. cordata et ' $A$. fordii sont toxiques. On pourrait cependant utiliser le premier pour I'engraissement du bétail, sauf pour les vaches, parce qu'il affectele goût et la consistance du beurre. Sa composition est la suivante :

Matières grasses. ............ 12

Matières' azotées............ 50

Extrait non azoté............ 19

Cellulose. ............... 3

Cendres .............. 7,5 


\section{Tourteau de Citrouille}

Les graines des citrouilles indigènes sont utilisées en diverses régions pour fabriquer une huile alimentaire. C'est le cas pour I'huile de «bereff» (Sénégal, Mauritanie). Le residu n'est pas un véritable tourteau, l'extraction se faisant par chauffage au-dessus d'eau bouillante. Il est riche en huile et surtout en azote, et devrait être donné avec des aliments hydrocarbonés. Il s'altere vite.

\section{Tourteau d'Arganea}

Après cuisson des graines de l'arbre à'Argan, Arganea sideroxylon, on obtient un tourteau qui est donné, dans le Sud marocain, aux vaches et aux chameaux. La saponine qu'il renferme (arganine) est peu toxique par la voie digestuve. Cependant, passant dans le lait, elle pourrait causer des accidents chez les enfants qui consomment ce dernier.

\section{Tourteau de Balanites ægyptiaca}

Les graines de cet arbre des régions subdésertiques sont traitées pour extraction de l'huile. Le tourteau n'a pas été utilisé. Il doit renfermer une saponine. Cependant, les animaux mangent le fruit entier sans inconvenient majeur (purgation). le tourteau a la composition suivante :

Matières grasses. ............ 6

Protéines ................ 48

Hydrates de carbone........... 30

Cellulose .................. 6

\section{Tourteau de Guizotia}

Guizotia abyssinica, G. oleifera, herbes parfois cultivées dans l'Inde et en Afrique orientale ont des graines riches en huile et fournissent un tourteau qui a été utilisé.

\section{Tourteau d'Hevea}

Les graines de l'arbre à caoutchouc, Hevea
Éasiliensis, fournissent, après extraction de l'huile, un tourteau qui aurait donné de bons résultats chez la chèvre et le mouton. Il renferme 43 à $44 \%$ d'hydrates de carbone et 30 à $34 \%$ de matières azotées.

\section{Tourteau d'Allanblackia}

Les noix d'Allanblackia stuhlmanni (Afrique orientale), après extraction de l'huile, donnent un tourteau qui renferme $14 \%$ de protéines et paut être donné aux bovins, mais avec circonspestion, en raison de sa teneur en tanin.

\section{Tourteau de Carthame}

Les graines de Carthamus indicus sont utilisées cans l'Inde pour l'obtention d'une huile alimentaire. Le tourteau a les qualités du tourteau de colza.

\section{Tourtean de Brassica eruca}

Cette crucifère fournit une huile très utilisée dans 1. Inde. Le tourteau est couramment distribué, surtout aux chameaux.

\section{Tourtean de Glyphea}

Glyphea laterifolia (Afrique tropicale) a une graine gui fournit de l'huile ressemblant à l'huile de coton. ie tourteau a sensiblement la valeur du tourteau de palmiste.

\section{Tourteau de Commiphora}

Le tourteau des graines de Commiphora zanziSarica est utilisé. Il renferme $6 \%$ o de graisses, $61,7 \%$ de protéines, $3,4 \%$ de cellulose et $13,2 \%$ d'sydrates de carbone.

\section{Graines oléagineuses diverses}

Diverses graines oléagineuses africaines donnent, apres exiraction de l'huile, des produits de valeur variable, dont la composition est la suivante.

\begin{tabular}{|c|c|c|c|c|c|c|}
\hline ESPECESS & ORIGINE & $\begin{array}{c}\text { EXTRAIT } \\
\text { éthéré }\end{array}$ & $\begin{array}{c}\text { ceLLLOSR } \\
\text { brute }\end{array}$ & $\begin{array}{c}\text { PROTÉINE } \\
\text { brute }\end{array}$ & $\begin{array}{c}\text { EXTRAIT } \\
\text { now L20te }\end{array}$ & CENDRES \\
\hline Poga oleosa..... & Afrique occidentale & $》$ & 9 & 41,51 & 40,74 & 8,75 \\
\hline Madia sativa ....... & Afrique du Sud & 0,55 & 26,14 & 30,19 & 28,81 & 6,2 \\
\hline Moringa aptera ..... & Égypte & 2,6 & 6,6 & 48,6 & 28 & 5,5 \\
\hline Moringa oleifera .... & Afrique occidentale & 8 & 3,96 & 52 & 20,2 & 3,84 \\
\hline Ochna pulchra............. & Rhodésie & 0,8 & 2,9 & 21,3 & 63,3 & 2,2 \\
\hline Ximenia americana...$\ldots \ldots \ldots$ & Afrique du Sud & 5 & 8,9 & 41,5 & 31,3 & 6,1 \\
\hline Pentaclethra macrophylla & Nigéria Sud & $\gg$ & 6,6 & 34,8 & 41,9 & 3,5 \\
\hline Pappea capensis ....... & Afrique du Sud & 0,4 & 5,1 & 38,3 & 42,9 & 5,9 \\
\hline Sterculia fœetida $\ldots \ldots \ldots$ & Gold Coast & 7,2 & 2,6 & 31,7 & 43,5 & 5,1 \\
\hline Trichodesma zeylanicum... & Tanganyika & 0,3 & 20,1 & 18,1 & 33,6 & 15,8 \\
\hline
\end{tabular}




\section{II. - RÉSIDUS \\ DE QUELQUES FRUITS TROPICAUX}

Ananas. - La partie externe du fruit, après épluchage de ce dernier pour l'extraction du jus ou la fabrication de conserves, est donnée aux animaux en remplacement du grain ou de la pulpe de betteraves. On la fait dessécher pour le transport. Quant aux pulpes, résidus de l'obtention du jus, on peut les faire dessécher ou les ensiler. La fermentation, dans ce dernier cas, donne un produit à odeur acidulée agréable, que les animaux acceptent volontiers sans troubles digestifs; il ne peut cependarsl suffire à assurer une ration d'entretien. La composition comparée de ces drèches en provenance d'Hawaï ou de Guinée française est la suivante :

\begin{tabular}{|c|c|c|}
\hline & $\begin{array}{l}\text { 'ANANAS } \\
\text { d'IIawäí }\end{array}$ & $\begin{array}{l}\text { ANANAS } \\
\text { de Guinée }\end{array}$ \\
\hline Matières grasses : & $\cdot 1,64$ & 4,44 \\
\hline Protéines $\ldots \ldots \ldots \ldots \ldots$ & 3,88 & 5 \\
\hline Extractif non azoté...$\ldots$ & 68,88 & 55,83 \\
\hline dont amidon ..... & 22,22 & $?$ \\
\hline Sucres............. & 22,22 & 9,83 \\
\hline Celluiose... & 22,22 & 30,83 \\
\hline Cendres......... & 3,33 & 3,88 \\
\hline
\end{tabular}

Les drèches peuvent être données aux bovins et aux porcs sans troubles digestifs, mais ne peuvent cependant suffire à fournir une ration d'entretien.

Bananes. - Les bananes vertes, non livrées au commerce, peuvent être réduites en farine; plus rarement, on a recours, pour l'alimentation du porc, à la farine industrielle; cette dernière, ou encore la banane séchée en fragments, a moins de valeur que la banane mûre, car elle ne peut guère être préparée qu'avec des fruits incomplètement mûrs. Elle renferme de la vitamine $B$, peu ou pas de vitamine $C$, et une diastase, l'amylase, que peut intervenir dans la digestion des hydrates de carbone.

La farine'de bananes vertes est pauvre en protéine $(4,9 \%)$. On l'a donnée aux volailles en remplacement partiel du maïs; cette substitution n'est pas favorable, même si on compense la pauvreté du produit par l'addition de soja ou de farine de poisson.
Les " peaux de bananes » provenant du traitement des fruits sont un aliment intéressant. Leur composition est la suivante :

\begin{tabular}{|c|c|}
\hline resèche & 16,2 \\
\hline Protéine & 6,1 \\
\hline Extrait éthéré & 8,7 \\
\hline Cellulose. .... & 10 \\
\hline Extrait non azoté. & 63,1 \\
\hline Sucre total & 22 \\
\hline Cendres ... & 11,7 \\
\hline
\end{tabular}

Café. - La pulpe de café, c'est-à-dire l'enveloppe charnue des graines, peut être utilisée fraiche ou sèche. Fraîche, elle s'altère rapidement. On peut l'ensiler. Sèche, elle peut être donnée poids pour poids en remplacement du mais ; mais elle est asssez difficilement acceptée quand on la donne seule: aussi la mélange-t-on à de la mélasse, à des feuilles séchées de bananier, ou encore à des fragments de tronc de bananier. Elle constituerait une bonne source de phosphore et de calcium.

Citrons et autres agrumes. - Dans les Indes occidentales, les résidus industriels du zeste et dc la pulpe de citron sont desséchés et utilisés pour l'alimentation des vaches laitieres. La farine obtenue aurait une valeur égale à celle de la farine de mais, en raison de sa teneur en matieres grasses et en protéine.

On peut aussi ensiler la pulpe de citron, seule ou avec une graminee (canne à sucre, natal grass). L'ensilage a la composition moyenne suivante (en Israël) :

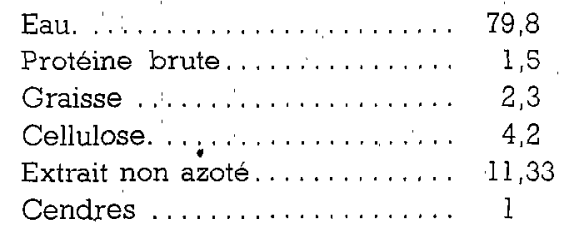

En Israël, on ensile également pour le bétail les marcs pressés provenant des oranges, des pamplemousses traités industriellement: Ces produits vont surtout aux vaches laitieres. Leur alimentation avec les citrons augmenterait la teneur, en matière grasse du lait, ce qui serait dû à l'enveloppe du fruit; mais le lait est teinté quand on donne la pulpe pressée deux heures avant la traite. 